\title{
La voz del consumidor en comunicación comercial: 40 años de evolución de la Planificación Estratégica publicitaria
}

Consumer voice in advertising: 40 years of Account Planning's development

Cristina Sánchez Blanco. Universidad de Navarra

Recibido: 4-XI-2010 - Aceptado: 29-XI-2010

Resumen:

La Planificación Estratégica en publicidad nació en 1968 gracias a dos publicitarios londinenses que vieron la necesidad de conocer a fondo al consumidor e integrarlo en el desarrollo de la publicidad y supuso una revolución en el mundo publicitario de aquella época. La publicidad, los medios de comunicación y el consumidor han sufrido cambios importantes por lo que el planificador también ha evolucionado para adaptarse. En este artículo se compara la situación de la publicidad en los años del origen de la disciplina y en la actualidad para entender la transformación del rol del planificador y su relevancia hoy en comunicación comercial.

Palabras clave:

Planificación Estratégica, publicidad, consumidor, investigación.

Abstract:

Account Planning was founded in 1968 by two English advertisers who saw the need to know deeply the consumer and integrate this knowledge into the advertising process. It was a revolution in the advertising world at that time. Advertising, media and consumer have undergone significant changes, so the planner has also evolved to adapt. This article compares the situation of advertising in the years of the origin of the discipline and now to understand the transformation of the role of the planner and its relevance today in advertising.

Keywords:

Account Planning, advertising, consumer, research. 


\section{Introducción}

La Planificación de Cuentas ${ }^{1}$ nació como disciplina publicitaria simultáneamente en dos agencias de publicidad londinenses, con el objetivo de que sus profesionales fueran embajadores de las personas, de los públicos, dentro de las agencias. Buscaba integrar la investigación en todo el proceso publicitario para comprender a fondo al consumidor y desarrollar el tipo de publicidad que realmente conectara con él. La idea que tenían las personas que lo desarrollaron era que si se incorpora al consumidor en el proceso se podrían conseguir unos objetivos claros y sería más fácil definir, y después medir, los resultados.

El objetivo de esta disciplina es conocer a fondo al consumidor e integrar ese conocimiento en el desarrollo de la publicidad. Supone, por tanto, elaborar un proceso para la gestión del conocimiento del consumidor. Las razones que llevaron al nacimiento de la disciplina en Londres en 1968 se debieron a causas relacionadas con la madurez del mercado publicitario y de la profesión publicitaria de aquella época.

En primer lugar, la madurez del mercado. El contexto de los años 50 estuvo protagonizado por el movimiento del consumidor y el auge del consumo, fruto de cambios sociales y culturales (McDonough, Egolf, 2003: 383). Entre dichas transformaciones destacó la entrada de la televisión, que provocó que se multiplicaran las opciones de comunicación con los consumidores. Esto llevó a las empresas a modernizar sus departamentos de marketing ya que se hacía evidente la necesidad de comprender al consumidor que estaba emergiendo para lograr comunicarse con él de otra manera. Ya no bastaba con una estrategia centrada en el producto. El consumidor era una parte activa y participante, y sus decisiones de compra estaban motivadas, entre otras causas, por la publicidad.

La segunda razón del origen de la Planificación de Cuentas tiene que ver con la madurez de la profesión publicitaria. Se entendió que el conocimiento del consumidor debía tener una repercusión en el mercado. Había que pasar a unos sistemas de trabajo que permitieran de verdad conseguir y evaluar la eficacia de la publicidad.

Así, en el principio de la Planificación de Cuentas encontramos dos escuelas de pensamiento lideradas por Stephen King en J. Walter Thompon (JWT) y Stanley Pollitt en Boase Massimi Pollitt (BMP). Con el mismo objetivo, la investigación profunda y la gestión de ese conocimiento del consumidor, King tenía una visión más cercana al marketing. Eso le llevó a crear el departamento de Planificación de Cuentas en JWT uniendo

1 Como se justificará más adelante, en su origen la disciplina se denominó Planificación de Cuentas por la traducción de Account Planning. Sin embargo, hoy se conoce por Planificación Estratégica y por ello es la denominación escogida para el título del artículo. 
a los profesionales que ya se encontraban en el de marketing y de medios para dar un servicio completo al anunciante basado totalmente en el consumidor (King, 1989: 37). Por su parte, la experiencia de Pollitt se centraba sobre todo en la investigación e intuyó la necesidad de un nuevo investigador que estuviera volcado en el consumidor para generar una buena publicidad a partir del servicio a los creativos. Este cambio le llevó a fundar una nueva agencia: BMP (Boase, Bullmore, Newman, 1998: 24).

El mercado mediático, publicitario y el consumidor han evolucionado mucho desde que nació la Planificación de Cuentas hace cuarenta años. Por tanto, como es obvio, el papel del planificador también ha cambiado para adaptarse (White, 2008: 22). Han trabajado siempre por conocer al consumidor pero ahora, los planificadores, deben adecuarse más aún a unos consumidores que tienen más poder (Rudder, 2001: 26). La Planificación de Cuentas es más importante que nunca porque el desarrollo de la era de la información ha hecho que crezca la necesidad de gestionar de forma integrada la información sobre el consumidor (Kelley, Jugenheimer, 2006: 10).

Tal y como se creó el planificador de cuentas para el modelo de marketing de los años 60, el siglo XXI demanda ahora un nuevo modelo de agencia, una capaz de ofrecer soluciones creadoras de valor a sus clientes donde la planificación de cuentas asume nuevas funciones ya que es una disciplina flexible que pone al consumidor en el centro del marketing respondiendo a las necesidades del negocio y del cliente (Zambardino, Goodfellow, 2003: 433).

Lo que nació de una manera modesta en dos agencias de publicidad en Londres se fue desarrollando hasta tal punto que le costó sólo diez años ser la norma común en las agencias de publicidad británicas. En la década de los 80, la Planificación de Cuentas ya era muy conocida y valorada por su contribución al desarrollo de una publicidad más efectiva que se podía vender mejor al cliente y por constituir una forma distinta de atraer nuevos negocios (Schofield, 1990: 61). En conclusión, contar en 1975 con un departamento de Planificación de Cuentas era extraño. En 1981 empezaba a estar de moda y ya en 1985 resultaba algo normal. En aquel momento casi ninguna agencia nueva empezaba sin el nuevo departamento y también comenzó a haber un importante desarrollo en las agencias ya establecidas (Bartle, 1985: 632). En pocos años, la Planificación de Cuentas se convirtió en una parte importante de las agencias de publicidad (Whelan, 1978: 39). El salto de la disciplina a Estados Unidos se produjo como consecuencia del desarrollo que tuvo en Reino Unido y constituyó el hito fundamental en la expansión de la Planificación de Cuentas. Allí, se introdujo gracias a una agencia pequeña asentada en California, Chiat/Day, en 1982. A partir de este momento se fue extendiendo a muchos países y hoy en día es la tónica general de las agencias de publicidad. 
Para entender bien qué funciones presenta el planificador hoy tenemos que hacernos cargo del contexto en el que nació la disciplina, qué cometidos desarrollaban sus profesionales y cómo la evolución del mercado, de los medios y del consumidor han hecho que, naturalmente, el planificador se adapte al nuevo entorno.

\section{Contexto de la evolución del planificador de cuentas}

En 1968 el conocimiento del consumidor comenzaba a ser una prioridad dentro de las agencias de publicidad, gracias al nacimiento de la Planificación de Cuentas. Ahora, nos damos cuenta de que es una exigencia dentro de cualquier empresa. Desde los años 90 se desarrolla la teoría de Integrated Marketing Communications (IMC o Comunicación Integrada de Marketing) que, poniendo al consumidor en el centro, vela para que los diferentes elementos de comunicación de marketing envíen un mensaje coherente al consumidor, para que la comunicación sea eficaz.

En concreto, en la evolución que ha sufrido la Planificación de Cuentas han influido en gran medida las transformaciones de los medios de comunicación, que, durante estos cuarenta años, han sido destacadas. Vamos a hacer un recorrido por el desarrollo de los medios para entender qué papel ha jugado el consumidor.

Antes del nacimiento de los medios masivos y desde los comienzos de la publicidad como una profesión en cuanto tal (desarrollada por los clásicos: Bernbach, Ogilvy, James Webb Young, entre otros) el papel que jugaban los medios de comunicación era decisivo para la publicidad. Para estos primeros profesionales, eran simples medios para alcanzar un fin, el consumidor. Por tanto, se consideraban iguales, no resaltaba ninguno por encima de otro y todo estaba dirigido por la relación personal con los consumidores. Es el momento de la afirmación: "El consumidor es tu mujer” de Ogilvy (Ogilvy, 1988: 96).

Sin embargo, el nacimiento de la televisión fue un acontecimiento importante. Los anunciantes se dieron cuenta de que sus mensajes podían llegar a mucha gente y el consumidor pasó a ser una audiencia homogénea y pasiva. En esa época, la planificación y la compra de medios se realizaban desde la propia agencia de publicidad. Hay que decir que no presentaba muchos problemas ya que las mayores inversiones se hacían en la televisión cuando la variedad de canales era escasa.

En esta situación, cuando comenzaba "el movimiento del consumidor", surge la Planificación de Cuentas, para volver a posicionar al consumidor en el centro del proceso de creación publicitaria, con dos visiones. Por un lado, Pollitt se dio cuenta de que era necesaria una persona que velara por los intereses del consumidor dentro de la agencia, y que fuera un nexo entre cuentas y creatividad, facilitando el salto creativo 
gracias a una investigación publicitaria centrada en el consumidor. Por su parte, King fue más allá y pensó ofrecer a los anunciantes una estrategia de comunicación de marketing basada en el consumidor. Por ello, decidió unir los departamentos de medios y de marketing de la agencia en el planificador de cuentas que se encargaría de la estrategia de medios y de marca, poniendo el foco en el consumidor.

El panorama empezó a cambiar cuando, en los años 70, los servicios de medios salieron de las agencias de publicidad por condicionamientos de tipo económico relacionados con la compra de espacio publicitario. Así, el fenómeno de la globalización del negocio publicitario, además de a las agencias y a los anunciantes, también afecta a las centrales de compra que por eso años comenzaban a formarse. Al principio eran media independents, empresas que compraban medios y trabajaban de manera autónoma. Fueron perdiendo esa independencia porque se empezaron a ligar a agencias o a anunciantes (Eguizábal, 1998: 426). Así, al desarrollarse ampliaron sus funciones hasta abarcar todas las actividades de medios, incluida la planificación y pasaron a llamarse agencia de medios (González Lobo, Carrero, 2006: 23). Por tanto, los anunciantes vieron que estas organizaciones tenían más capacidad de negociación con los medios y les podían ofrecer mejores precios.

De esta manera, el planificador de cuentas se quedó en la agencia encargado únicamente de enfocar el salto creativo y desarrollar la estrategia publicitaria, inspirando ideas creativas con un pensamiento innovador (Collin, 2003: 442). De hecho, ésta es la razón por la que la escuela que se exportó desde Reino Unido en primer lugar a Estados Unidos fuera la de Pollitt con una clara visión creativa. Por su parte, los planificadores de medios fueron creciendo en influencia como consejeros de las estrategias de comunicación de marketing de los clientes. Esto se debía, en gran medida, a la necesidad de incrementar sus ingresos vendiendo servicios de investigación adicionales (Collin, 2003: 443).

Lo que hemos visto hasta aquí se engloba en la disciplina de la publicidad convencional, porque no será hasta los años 80 cuando empiecen a desarrollarse nuevas disciplinas para llegar a los públicos: las promociones, el marketing directo, los patrocinios, las relaciones públicas, etc. que comenzaron a formar parte de los planes de marketing de los clientes de la agencia (Victoria Mas, 2005: 42-43).

A esto se añade la fragmentación mediática y la segmentación de las audiencias, la existencia de un consumidor más maduro, más informado y más activo y la baja efectividad de la publicidad convencional, sobre todo de la televisión. De esta manera, emergen y se desarrollan las teorías de la comunicación integrada de marketing que hablan de la integración de las diferentes herramientas de comunicación para enviar un mensaje coherente al consumidor y alcanzar una comunicación más efectiva. En concreto, los anunciantes quieren una rentabilidad mayor y se entiende que busquen alternativas de comunicación para llegar de 
un modo eficaz a los consumidores invirtiendo más en medios no convencionales, como marketing directo o Internet. A partir de los años 80 se comienza a hablar de los mercados transnacionales. La historia del fenómeno de concentraciones y de la globalización había empezado en los años 70 (Eguizábal, 1998: 418).

En este sentido, los cambios radicales en la naturaleza de los medios, la tecnología y la sociedad demandan un comportamiento diferente en el proceso de la planificación de la comunicación. En la actualidad vemos que cada vez el consumidor es más “poderoso" (McIlrach, 2002: 17) y los anunciantes tienen que innovar en las formas para llamar su atención, y mantener con ellos una relación. Se necesita un nuevo planificador de cuentas que esté centrado en el consumidor y no en los medios. Por ello, el planificador de cuentas renace de manera adaptada a esta nueva situación.

\section{Funciones del planificador de cuentas actual}

Con el objetivo de comprender las peculiaridades que presenta el planificador hoy en día, comenzaremos haciendo una comparativa entre las funciones que corresponden a la filosofía original de la Planificación de Cuentas y las que presenta hoy en día. Nos basamos, en primer lugar, en las definiciones que Account Planning Group de Reino Unido ha publicado: la primera es de 1986 y recoge todas las ideas de los primeros profesionales, después se hizo una actualización en 2001 y otra en 2007, a las puertas del 40 aniversario del nacimiento de la disciplina, que se cumplió en 2008.

Introducimos una tabla (Tabla I) donde se mencionan las diferentes funciones del planificador, comparando las que ha tenido tradicionalmente y sigue teniendo y las que está incorporando desde los últimos años como consecuencia de la evolución de la profesión. Las exponemos según cuatro categorías, siguiendo a Baskin y a Pickton: investigación, voz del mercado, estrategia y cooperación en la creatividad (Baskin, Pickton, 2003: 420-423). De las dos escuelas que motivaron el origen de la disciplina en el desarrollo de la disciplina tuvo más relevancia la escuela de Stanley Pollitt (en la agencia Boase Massimi Pollitt, BMP). La estructura que planteaba resultaba fácil de adaptar a las agencias pequeñas donde el trabajo gira en torno a una persona, el planificador, que se involucra y se integra en el trabajo de toda la agencia. Ejemplo de esto es la entrada en Estados Unidos en 1982 de la mano de Chiat/Day, una agencia con características similares a BMP, que se identificó plenamente con los principios sobre la importancia del conocimiento del consumidor (Stabiner, 1993: 47).

Sin embargo, la que ahora emerge con más claridad y cobra más importancia es la de Stephen King (J. Walter Thompson). Este profesional innovó un planteamiento estratégico al unir a las personas dedicadas a la 
planificación de medios y a las de marketing en el planificador estratégico. Este nuevo perfil se ocupaba de la estrategia (de medios y de marca) basada en un conocimiento profundo del consumidor (Bond, Kirshenbaum, 1998: 166-167). Al detenernos en la tabla, se comprende cómo prima el factor estratégico, aspecto en el que King incidió más y que es la categoría en la que se han añadido más funciones. Con el crecimiento y la sofisticación en el mercado y de la marca, la fragmentación de los medios y el énfasis en la comunicación integrada, el planificador de cuentas ya no es sólo la voz del consumidor sino también la voz del mercado, término que recoge toda esta evolución (Baskin, Pickton, 2003: 422).

Tabla I. Comparación funciones del planificador de cuentas. Elaboración propia²

\begin{tabular}{|lll|}
\hline & TRADICIONALES & NUEVAS \\
\hline INVESTIGACIÓN & Investigador de mercados. & Centro intelectual de la agencia. \\
& Analista de datos. & \\
& Moderador de grupos cualitativos. & \\
& Centro de información. & \\
& Generador de conocimiento a través de & \\
& las tendencias que marca el mercado y & \\
& anticipador de movimientos sociales & \\
& (futurólogo). Antropólogo. & \\
\hline VOZ DEL MERCADO & Voz del consumidor. & Voz y posicionamiento de la \\
& & marca. \\
\hline ESTRATEGIA & Pensador estratégico. & Planificador de medios. \\
& Creador de la estrategia. & Planificador de comunicación. \\
& Consultor en el desarrollo de nuevos del proceso de integración \\
& productos de una marca. & Líder dratégico. \\
& Descubridor de insights. & Comunicación multidisciplinar \\
& Pensador polémico. & (IMC). \\
\hline COOPERACIÓN & Responsable del brief creativo. & \\
CREATIVIDAD & Utiliza el conocimiento para que sirva a & \\
& los creativos. & \\
& Moderador de brainstorming. & \\
\hline
\end{tabular}

2 Tabla elaborada a partir de las siguientes fuentes: Baskin, Pickton, 2003; Baskin, 2007; Hackley, 2003; Weichselbaum, 2008; Kelley, Jugenheimer, 2006; Mikes, 2007; Lannon, Baskin, 2007; Do Amaral, 2004. 
Por eso, el planificador de cuentas actual tiene nuevos cometidos:

- Paso de ser un planificador de cuentas a ser un planificador estratégico.

- Planificador de la comunicación:

- Papel activo en la planificación de medios.

- Guardián de la marca.

- Investigación global sobre el consumidor.

- Especialización: ámbito de actuación en otras disciplinas.

Vamos a desarrollar estas funciones una por una.

\subsection{De planificador de cuentas a planificador estratégico}

La Planificación de Cuentas nació en el entorno de las agencias de publicidad con el objetivo de que una persona preparada se ocupara de la investigación en profundidad sobre el consumidor y el mercado para integrar ese conocimiento en toda la agencia. Así, era la conciencia del director de la cuenta (Pollitt, 2000: 4) y cooperaba con el trabajo de los creativos para obtener una publicidad eficaz. De esta manera, se ideó el nombre de la nueva figura que iba a ser una mezcla de la persona de cuentas y la de medios, por lo que se llamó account planner, que traducimos por planificador de cuentas.

Por tanto, en la época de Pollitt y de King, las agencias pasaron de ser consejeras de marketing generalistas a ser especialistas en publicidad y los planificadores emergieron para ayudar a hacer una publicidad creativa más relevante para los consumidores y más fiable y rentable para los clientes. En contraposición, hoy, en un entorno de marketing integrado, las agencias van moviéndose hacia lo más general, hacia ideas de marketing y de comunicación multidisciplinar. En este sentido, el planificador amplía su margen y tiene un gran potencial para la planificación y la gestión de IMC (Baskin, Pickton, 2003: 424) que le hace el líder del proceso de integración estratégico, responsable de obtener lo mejor de los equipos especializados en cada herramienta de comunicación (Lukas, Walker, 2008: 279). Su conocimiento profundo sobre el consumidor le hace capaz de saber dónde y a través de qué tipo de comunicación se le puede encontrar con eficacia. De esta manera, tiene más sentido y más fuerza que ahora se denominen planificadores estratégicos, nombre que en muchas ocasiones siempre han adoptado sobre todo en la práctica. Por ello, desde ahora vamos a referirnos a ellos como planificadores estratégicos. Así, para Baskin un nombre más acertado que account planner es brand strategist, porque describe más la función que realiza (Baskin, 2006: 81). 
En concreto, con el desarrollo de la sociedad de la información y la fragmentación de los medios, los planificadores han pasado a ser planificadores de comunicación. Coordinan estratégicamente la marca a través de los diferentes tipos de comunicación de marketing y conocen todas las habilidades de las diferentes disciplinas y cómo se accede al consumidor a través de ellas. En este entorno, se entiende que el papel del planificador sea indispensable porque controla la visión central de la marca que se tiene que transmitir en cada tipo de comunicación (Block, 1994: 24-25). Los planificadores, así, se encargan de la comunicación total de la marca (Broadbent, 1995).

\subsection{Planificador de comunicación}

Los cambios radicales en la naturaleza de los medios, la tecnología y la sociedad demandan un comportamiento diferente en el proceso de la planificación de la comunicación. Por eso, en la industria, de manos de los planificadores estratégicos ingleses, nació en 2002 un nuevo término (Saunders, 2004: 38): Media Neutral Planning que se sitúa como la nueva gran tendencia en la comunicación de marketing (Clifton, 2002). Como matiza Tapp, los últimos años lo han visto como lo más actual (Tapp, 2005: 133). De hecho, a partir de la publicación del libro The Communications challenge, a practical guide to media neutral planning en 2003 por la Asociación Profesional de Planificación Estratégica de Reino Unido (APG UK) y de una serie de artículos sobre la materia, los planificadores estratégicos comenzaron a hablar de la neutralidad mediática.

Esta planificación de medios neutral es lo que Taylor denomina una nueva Planificación de la Comunicación, que será la gran catalizadora del cambio en el futuro y significa planificar el uso de la comunicación a través de todos los canales y disciplinas de marketing a partir de la selección de los consumidores idóneos para que se relacionen con la marca (Taylor, 2005: 5-7). Es una planificación, por tanto, que tiene una perspectiva de $360^{\circ}$ donde se entiende que lo esencial es el consumidor. No se pretende emplear algunos medios más que otros por motivos económicos, como se hacía anteriormente, sobre todo, con el uso de la televisión que es la que reportaba más dinero. Se trata, por tanto, de desarrollar un plan holístico, a través de las funciones de marketing, que define cómo la marca se comunicará con sus consumidores. Ante la fragmentación mediática, se multiplican las maneras para conectar con los consumidores y una planificación estratégica del mix de medios proporcionará una comunicación efectiva con los consumidores (Crosier, Grant, Gilmore, 2003: 4).

En este epígrafe vamos a ver en qué consiste esta planificación de la comunicación enfocada en el consumidor que implica que el planificador participa de la estrategia de marca y de medios, es la voz del mercado y el guardián de la marca y realiza una investigación global sobre el consumidor. 


\subsubsection{Papel activo en la planificación de medios}

La planificación de la comunicación necesaria hoy es la unión de la planificación estratégica y la planificación de medios, y el que más sentido tiene que la desarrolle es el planificador estratégico. Tungate señala que, aunque hoy en día no hay casi ninguna agencia que tenga un departamento de medios, en el entorno de medios convergentes, es imposible separar estas disciplinas. Los anunciantes están demandando campañas multimedia y esto requiere grandes habilidades en la planificación de medios, y un trabajo conjunto de creatividad y de medios. Sostiene que parece que haber dejado que los medios se fueran de las agencias fue un error y, en este sentido, hay iniciativas de agencias que quieren unir los medios y la creatividad (Tungate, 2007: 155-161). Esto se entiende porque, como establece Feldwick, separar la creatividad y los medios "no es necesario ni deseable. Ambos aspectos dependen de entender al mismo consumidor" (Feldwick, 1992: 37).

Hoy en día las empresas exigen un perfil que tenga habilidades de planificación de medios y de planificación de la marca. Así, vemos cómo el planificador estratégico tradicional se convierte en un brand/media/ communications planner (Zambardino, Goodfellow, 2003: 432).

Sánchez Hermida señala que, aunque se originó en las agencias creativas, “actualmente, el planning, en un contexto de mercado cada vez más complejo, es una pieza fundamental en el armado de la estrategia de medios" (Sánchez Hermida, 2006: 231). Esto se entiende por varias razones:

- $\quad$ El planificador es el nexo entre la planificación de la comunicación y la planificación de medios porque el posicionamiento de la marca no tendrá éxito si no está apoyado adecuadamente en la selección y el emplazamiento de los medios. Las opciones de medios están fragmentándose, y cada vez más, presentan una oportunidad y una exigencia para unir la estrategia de marca con la selección de medios. El planificador estratégico debe poder decidir sobre el plan de medios para crear sinergias con el posicionamiento de la marca (Kelley, Jugenheimer, 2006: 7-10).

- Es vital enlazar la comprensión del consumidor con la de los medios, asunto del que se tiene que ocupar el planificador estratégico (Beale, 2003: 2). De hecho, están surgiendo nuevos planificadores estratégicos que combinan estas dos disciplinas (Crosier, Grant, Gilmore, 2003: 13) ya que la planificación de medios se acerca mucho y llega a incluirse dentro de la Planificación Estratégica (Jones, 1968: 375). Se une el trabajo de los planificadores estratégicos con el de los planificadores de medios con el fin de crear un vínculo estratégico común (Tilley, 2000: 193-211), por lo que cada vez es más importante que los planificadores de medios y los de cuentas trabajen juntos (O’ Donoghue, 1997: 121). 
- $\quad$ El papel del planificador estratégico en la estrategia publicitaria de medios es ayudar al equipo de medios a analizar cómo puede unir mejor al consumidor con la marca. El planificador estratégico conoce al consumidor y sabe en qué momento está más receptivo para un mensaje en concreto (Kelley, Jugenheimer, 2006: 104-106).

- $\quad$ Con todo, la comprensión del consumidor y de su respuesta (fruto de la investigación del planificador estratégico) facilita la planificación de los medios.

La integración de los diversos medios se constituye, hoy en día, en la estrategia de crecimiento de las agencias; y una de sus misiones es el conocimiento de cómo los consumidores reciben la comunicación. Cada vez es más importante para los planificadores estratégicos entender las posibilidades de cada medio, por público objetivo y por categoría, y conocer cómo y cuándo deben ser utilizados para alcanzar los objetivos de la marca (Baskin, 2001). Los planificadores estratégicos deben combinar las dos disciplinas: account planning y media planning y así, se les puede llamar communications planners (Crosier, Grant, Gilmore, 2003: 13).

Los planes de medios tienen que dirigirse más a la audiencia, deben estar personalizados según sus necesidades y tienen que ser eficientes en cuanto a costes (Shaver, 1995: 29). Coordinar el mensaje de la marca con los canales de comunicación en todos los puntos de contacto de ésta con el consumidor ayudará a mejorar la eficacia de la comunicación, y en consecuencia, el retorno de la inversión (Grimes, 2004: 53-54). Una marca que tiene coherencia de identidad, de mensaje y de voz es más propensa a dejar una imagen positiva en la mente de los consumidores. Así, los planificadores estratégicos deben velar por la coherencia de la marca a través de cualquier tipo de comunicación en un entorno integrado.

\subsubsection{Guardián de la marca}

Hoy en día los anunciantes buscan en el trabajo de las agencias más rentabilidad de su comunicación por lo que es decisiva la eficacia. El planificador estratégico trabaja para que la empresa se pueda diferenciar de sus competidores y la única forma en la actualidad es a través de las marcas (Crosier, Grant, Gilmore, 2003: 4). Siempre se han ocupado de las marcas, pero hoy en día adquiere más importancia porque es más difícil llegar a los consumidores (Goodlad, 2008: 20). En este sentido, los planificadores estratégicos construyen y mantienen la equidad de la marca a través de una comunicación consistente en los valores de la marca. Además, la proliferación de marcas, la fragmentación de los medios, el aumento de la competencia y de los costes de introducción de nuevos productos y una mayor responsabilidad por la rentabilidad han ocasionado que las empresas reparen en el valor de las marcas (Keller, 1998: 31-34). 
La Planificación Estratégica trabaja para el posicionamiento de la marca y por ello, es la disciplina más apropiada para unir todos los elementos de la comunicación de la marca en su posicionamiento. Siendo el abogado del consumidor, el planificador estratégico está involucrado en todas las facetas de la estrategia de la marca y su reflejo en la comunicación de marketing. Quizá la decisión más importante en marketing es cómo estará posicionada la marca para que sea única para el consumidor. Y, para que sea efectivo, hay que trabajar por unir los objetivos del negocio con cómo ven los consumidores la marca. De esta manera, trasladar la estrategia de posicionamiento a la estrategia de publicidad y de medios es vital para la marca. Así, el planificador estratégico es el encargado de posicionar la marca a través de todas las formas de comunicación y que ese posicionamiento se dé internamente en todos los empleados de la empresa y externamente con todos los consumidores. La Planificación Estratégica puede ser un trabajo o un departamento, o un proceso en una agencia de publicidad o en un grupo de marketing pero lo importante es que se ocupa de la marca (Kelley, Jugenheimer, 2006: 3).

Muchos planificadores se han posicionado como "gente de marcas" (Lukas, Walker, 2008: 281) hasta el punto de que se les denomina guardianes de la marca (Hackley, 2003: 452) porque ayudan a la empresa a cultivar su personalidad de marca (Kelley, Jugenheimer, 2006: 64). En un mercado maduro donde las marcas se diferencian difícilmente, tiene una labor importante el planificador "al ser un especialista en la marca y su desarrollo, buscando nuevas estrategias que delimiten la labor de la comunicación en una línea lógica con el producto y la empresa" (Etxebarría, 2005: 11-119).

Siguiendo a Ollé, se ha pasado de la Unique Selling Proposal (USP) al Branding porque "gestionar una marca en la actualidad es ser capaz de vincular una serie de significados o valores a nuestra oferta" (Ollé, 2005: 122). La USP es el concepto racional únicamente y Branding lo une con los valores emocionales de la marca. De hecho, hoy en día las marcas se diferencian cada vez menos unas de otras (The Commoditization of Brands) por lo que es necesario tener una estrategia de marca para que "tenga un poder de atracción muy claro, un conjunto de significados relevantes e incuestionables que consigan un espacio no sólo en la cabeza sino en el corazón de nuestros consumidores" (Ollé, 2005: 124). En este sentido, para obtener la atención de los consumidores, las marcas tienen que involucrar a los consumidores a través de acciones basadas en la experiencia (Gonsalves, 2008: 20). Una marca fuerte tiene que informar, diferenciarse y seducir y para ello es necesario un buen gestor de marcas, que es el planificador estratégico (Ollé, 2005: 124-125). La Planificación Estratégica es más importante que nunca por todo lo que tienen que hacer las marcas para prosperar (Collin, 2010: 22). Y para llevar esto a cabo es necesario que el planificador estratégico desarrolle una investigación completa sobre el consumidor. 


\subsubsection{Investigador global sobre el consumidor}

Los planes de medios deben basarse en una exhaustiva comprensión de la relación entre el consumidor, la marca y las opciones mediáticas potenciales (O’ Regan, 2003: 291). Así, se identifica la manera más efectiva de conectar con el consumidor a partir de una investigación profunda sobre él como persona, de manera holística y sobre el uso que tiene de los medios (Taylor, 2005: 10). Por tanto, es necesario considerar todos los puntos potenciales en los que las marcas influyen en su vida. En el mundo real, los consumidores no existen en medios específicos, cada target consume una combinación de medios dependiente del acceso o la relevancia del mensaje que se está transmitiendo. Esto requiere algo más que únicamente generar una fotografía de quiénes son, sus ideas, su consumo de medios y su relación con la empresa.

Una de las mejoras más notorias en la relación entre los oferentes y los demandantes es la centralidad del consumidor a la que se ha llegado. Los valores de las marcas deben comunicarse a los consumidores en cada punto de encuentro para adquirir y retener consumidores valiosos.

En definitiva, como señala Powers al resumir las ideas del congreso sobre Planificación Estratégica organizado por la Asociación Americana de Agencias de Publicidad (AAAA) en julio de 2008, los planificadores se tienen que involucrar en el mercado, en las casas de los consumidores, en sus mentes, en sus vidas, en sus culturas locales y globales e influir en ellos con buenas ideas que les hagan conectar con las marcas (Powers, 2008). El planner debe introducirse en el proceso mediático y analizar las tendencias que se ven (Connolly, 2008: 37), conocer al consumidor en todos los ámbitos, tanto en las actitudes ante determinadas categorías o marcas, como también en cómo emplean su tiempo y qué otras cosas de sus respectivas vidas llaman su atención (Morgan, 2002).

Comprender los procesos de los consumidores es fundamental para la planificación de la comunicación y para saber cuál es la comunicación que intercepta a las personas en los momentos críticos de decisión. Estas consideraciones han desembocado en el incremento de la investigación directa, basada en la observación del consumidor y su comportamiento, tanto dentro de las tiendas (compra en compañía) o en sus casas y comunidades (etnografía). Aunque éstas no revelan la forma de pensar de los consumidores, se acercan a la realidad de las personas en el uso de marcas y de los medios que realizan (White, 2004: 39).

Estas funciones que venimos explicando se concretan en el día a día en que el planificador las ejerce no sólo en las agencias de publicidad, su ámbito de origen. Así, posiciona la marca a través de todos los tipos de comunicación y su trabajo relacionado con el descubrimiento y análisis de los insights sobre el consumidor sirve para cualquier herramienta de comunicación. En el siguiente epígrafe, vamos a considerar cómo el planificador trabaja en otras áreas, independientes de la publicidad convencional. 


\subsection{Nuevas disciplinas donde el planificador desempeña su trabajo}

La Planificación Estratégica ha evolucionado con la naturaleza cambiante del negocio publicitario. Ahora, las agencias ofrecen a sus clientes una comunicación integrada y simultáneamente los planificadores estratégicos han adoptado nuevos papeles. Se han especializado más y han empezado a trabajar en otras organizaciones. Las agencias se han convertido en globales para reflejar las necesidades de sus clientes (Weichselbaum, 2008: 255). Los planificadores, en este sentido, ya no piensan en términos de publicidad, sino de comunicación (Broadbent, 1995: 27).

En 2008 se han celebrado los cuarenta años de su nacimiento y la Planificación Estratégica ha llegado a ser una función que existe no sólo en las agencias de publicidad sino también en los departamentos de marketing de los anunciantes, en agencias de marketing directo, en consultoras de diseño, de marca, de relaciones públicas, agencias de medios, etc. (Baskin, 2008: 39). En los últimos diez años la mayoría de las agencias de marketing directo, promoción de ventas o de diseño y de consultoría (White, 2008: 23) más relevantes han empezado a tener un departamento de planificación u ofrecen directamente sus servicios (Griffiths, 2002: 45).

El planificador estratégico trabaja para que surja una gran idea que pueda dirigir la estrategia de marca a través de todas las formas de comunicación, externamente para los consumidores e internamente para los empleados (Ryan, Montague, 2007: 143). Al participar en otras áreas además de la publicidad, los planificadores aportan valor de manera diferente porque se han especializado. Griffiths señala seis tipos de planners (Griffiths, 2008: 24):

- $\quad$ Brand planners: se encargan de tareas relacionadas con la reputación de la marca.

- $\quad$ Engagement planners: se tienen que preocupar de que los consumidores se involucren con la marca, en un entorno donde lo primero es conseguir su atención.

- Interactive planners: adquieren funciones, gracias a la evolución tecnológica, en la comunicación interactiva.

- Channel/connection planners: analizan los medios y canales de comunicación para establecer una relación con los consumidores.

- Data planners: usan eficazmente las bases de datos (Customer Relationship Management).

- $\quad$ Content planners: tienen un nuevo papel gracias a que los consumidores emplean mucho tiempo creando y consumiendo contenido generado por otros (user generated content) y los profesionales del marketing deben pensar cómo aprovecharlo. 
El papel tradicional de representante del consumidor para ayudar en la agencia a producir los mejores anuncios de televisión, ha dado paso a participar en todas las formas de comunicación y a que el planificador busque al consumidor en cualquier actividad donde ellos pasen el tiempo (Cuneo, 2007: 12). En este sentido, García defiende que, al encontrarnos en la era del entretenimiento, el consumidor está en todas partes y, por lo tanto, las marcas compiten por su atención en cualquier actividad de ocio: un partido de fútbol, un videojuego, una red social, etc. y la función del planificador es saber dónde encontrarles (García, 2008: 59).

\section{Conclusiones}

Hoy en día el centro de la comunicación de marketing es el consumidor. Con la continua evolución e hibridación de los existentes canales mediáticos, desde la perspectiva de la planificación, el consumidor es la clave para el proceso. En este contexto, el planificador estratégico en publicidad es el integrador de los diferentes elementos de comunicación de marketing para que el anunciante mande una comunicación integrada al consumidor porque es el que le conoce en profundidad. La gestión del conocimiento del consumidor es esencial en la actualidad y la Planificación Estratégica publicitaria es la única sistematización que se ha desarrollado y está siendo útil en comunicación comercial, a través de una visión estratégica de la relación entre la marca, los medios y el consumidor.

Actualmente las empresas exigen un perfil que recoja la planificación de medios y la planificación de la marca, esto es, una planificación de la comunicación enfocada en el consumidor. Por el desarrollo del consumidor y de los medios es necesario alcanzarle a través de muy diferentes puntos de contacto por lo que hay que conocerle en profundidad. El planificador estratégico es el encargado de realizar una investigación global sobre el consumidor actual.

El conocimiento del consumidor debe significar una comprensión genuina y proactiva de lo que el mercado necesita realmente. Hoy en día, el reto de los negocios para los anunciantes y para las agencias es cómo conocer y tratar las necesidades de los consumidores.

En síntesis, el planificador estratégico participa activamente en que la comunicación actual se centre en el consumidor y que, de esta manera, sea eficaz y rentable para los anunciantes. Trabaja para tener una relación con el consumidor con estas características:

- $\quad$ Conoce en profundidad al consumidor como una persona completa.

- $\quad$ Realiza estrategias de invitación. 
- $\quad$ Mantiene una relación sostenida en el tiempo.

- Involucra al consumidor con la compañía, quiere su fidelización, personaliza la relación.

- $\quad$ Busca su compromiso.

- Capta su atención.

Partiendo de una investigación global sobre el consumidor, el planificador realiza una gestión integrada de dicho conocimiento con una visión estratégica. Con ello, trabaja para posicionar la marca de forma exclusiva en la mente del consumidor, utilizando todas las formas de comunicación.

En un entorno integrado como el actual, los anunciantes demandan eficacia y rentabilidad, y para ello el planificador estratégico busca al consumidor y une los objetivos de medios y de la marca en una planificación de la comunicación que tiene como foco al consumidor. De esta manera, la Planificación Estratégica publicitaria es una disciplina flexible que pone al consumidor en el centro del marketing y responde a las necesidades del negocio y de los anunciantes y cada vez más vemos cómo se amplían las empresas donde los planificadores pueden trabajar y se van especializando en nuevas áreas.

\section{Referencias bibliográficas}

Bartle, J. (1985): “Account Planning: has it a future?”, Admap, December, pp. 632-633.

Baskin, M. (2001): What is account planning and what do account planners do exactly? A revised millennium definition. APG London. Disponible http://www.apg.org.uk/about-us/what-is-planning.cfm. (Fecha de consulta 15/10/10).

(2006): "Planning: actividad emergente. Bucear en la mente de los consumidores", Mercado, Septiembre, pp. 80-82.

(2008): "A bit of update”, en Weichselbaum, H. (ed.): Readings in Account Planning. Chicago: The Copy Workshop.

Baskin, M., Pickton, D., (2003): “Account Planning: from genesis to revelation”, Marketing Intelligence and Planning, 21, 7, pp. 416-424.

Beale, C. (2003): “Planning principles should be protected from the interlopers”, Campaign, 29 August, p. 2.

Block, R.W. (1994): “Advertising in suspense: the future of planning. Part 5: the year is 2010", Campaing, March 25, pp. 24-25.

Boase, M., Bullmore, P., Newman, J. (1998): “Planning 30 years on”, Campaign, March 25, p. 24.

Bond, J., Kirshenbaum, R. (1998): Under the radar: talking to today's cynical consumer. UK: John Wiley \& Sons.

Broadbent, T. (1995): “Recession may be the best thing to happen to planners", Campaing, June 23. 
Clifton, A. (2002): "Media-neutral planning -what is it?", Report, Campaign, $8^{\text {th }}$ November. Disponible en: http://www.apg.org.uk/publications/medianeutralarticles/mnp2_cliftonanthony.cfm (Fecha de consulta 19/12/08).

Collin, W. (2003): “The interface between account planning and media planning -a practitioner perspective”, Marketing Intelligence and Planning, 21, 7, pp. 440-445.

Collin, W., Hatton, R., Morgan, A., Owen, J., Watson, S. (2010): “The future of planning. Admap planner's roundtable”, Admap, February, pp. 20-26.

Connolly, C. (2008): “Communications planning in the 21st century”, Admap, September, pp. 37-38.

Crosier, K., Grant, I., Gilmore, C. (2003): "Account Planning in Scottish advertising agencies: a discipline in translation”, Journal of Marketing Communication, 9, 1, pp. 1-15.

Cuneo, A. (2007): “Account planners at a crossroads”, Advertising Age, July 30, pp. 12-14.

Do Amaral, R. (2004): O papel do Planejamento de Comunicaçao e o contexto brasileiro. Disponible en: http://www.bebadosobrio.org/Papel\%20do\%20planejamento\%20de\%20comunicacao\%20(2004).pdf (Fecha de consulta 12/11/08).

Eguizábal, R. (1998): Historia de la publicidad. Madrid: Eresma \& Celeste.

Etxebarría, J.A. (2005): “Las empresas publicitarias”, en Victoria Mas, J.S. (coord.): Reestructuras del sistema publicitario. Barcelona: Ariel Comunicación, pp. 104-119.

Feldwick, P. (1992): “Coherence and fragmentation”, Admap, May, pp. 36-37.

García, C. (2008): El libro de Bob. La nueva publicidad del siglo XXI. Madrid: Zapping/ M\&C Saatchi.

Gonsalves, J., Goodlad, N., Sinnock, M., Murray-Burton, G., Murphy, G. (2008): “The future of planning”, Campaing, July 18, pp. 20-21.

González Lobo, M.A., Carrero, E. (2006): Manual de planificación de medios, 4a edición. Madrid: ESIC Editorial.

Griffiths, J. (2002): “Above and beyond advertising planning”, Admap, February, pp. 45-46.

(2008): “Where to next? Account Planning at 40", Admap, April, pp. 24-25.

Grimes, J. (2004): “Creative inspiration”, en Saunders, J. (ed.): The Communications Challenge: a practical guide to media neutral planning. London: The Account Planning Group. pp. 53-80.

Hackley, C. (2003): "From consumer insight to advertising strategy: the account planner's integrative role in creative advertising development", Marketing Intelligence and Planning, 21, 7, pp. 446-452. http://www.apg.org.uk/publications/ medianeutralarticles.cfm (Fecha de consulta 19/12/08).

Jones, R.W. (1968): “Are media departments out of date?”, Admap, September, pp. 375-376.

Keller, K.L. (1998): Strategic Brand Management. Building, measuring and managing brand equity. New Jersey: Prentice Hall. 
Kelley, L.D., Jugenheimer, D.W. (2006): Advertising Account Planning. A practical guide. New York: M.E. Sharpe.

King, S. (1989): "The anatomy of Account Planning”, Admap, November, pp. 36-40.

Lannon, J., Baskin, M. (Ed.) (2007): A master class in brand planning. The timeless works of Stephen King. West Sussex: John Wiley \& Sons, Ltd.

Lukas, S., Walker, S. (2008): "Your next job description: what clients think", Weichselbaum, H. (ed.): Readings in Account Planning. Chicago: The Copy Workshop.

McDonald, M., Wilson, H. (2002): The new marketing. Transforming the corporate future. Oxford: Butterworth Heinemann.

Mc Donough, J., Egolf, K. (2003) (Ed.): The Advertising Age Encyclopedia of Advertising, 1 (A-E). NY: Taylor \& Francis Group, voz "Consumer movement".

McIlrach, S. (2002): "A new creative revolution”, Admap, September, pp. 17-18.

Mikes, A. (2007): Turn account executives into account planners. Wyomissing, PA: Second Wind Ltd.

Morgan, R. (2002): “Media-neutral planning -what is it?", Report, Campaign, $8^{\text {th }}$ November. Disponible en: http://www.apg.org.uk/publications/medianeutralarticles/mnp2_morganrebecca (Fecha de consulta 11/08/08).

O’Donoghue, D. (1997): “Account planning and media planning”, en Cooper, A. (Ed.): How to plan advertising, London: Cassell-APG, pp. 121-136.

O’Regan, M. (2003): “Editorial: Media neutral planning -a new role for marketing databases?”, Journal of Database Marketing, 10, 4, pp. 291-292.

Ogilvy, D. (1988): Confessions of an advertising man. New York: Atheneum.

Ollé, R. (2005): “El planner: la voz del consumidor en la agencia”, en Fernández Gómez, J.D. (coord.): Aproximaciones a la estructura de la publicidad. Desarrollo y funciones de la actividad publicitaria. Sevilla: Comunicación Social Ediciones y Publicaciones, pp. 115-131.

Pollitt, S. (2000): Pollitt on planning. Three papers by Stanley Pollitt. London: Admap Publications.

Powers, S. (2008): "What you missed at last at week's 4 A's Planning Conference", Advertising Age, July 29. Disponible en: http://adage.com/agencynews/article?article_id=129983 (Fecha de consulta 11/08/08).

Rudder, S. (2001): “What future for ad agencies?", Admap, May, pp. 26-27.

Ryan, R., Montague, T. (2007): “In pursuit of an intense response”, en Lannon, J., Baskin, M. (Ed.): A master class in brand planning. The timeless works of Stephen King, West Sussex: John Wiley \& Sons Ltd, pp. 141-143.

Sánchez Hermida, J. (2006): “Planning en Latinoamericano más allá de la publicidad”, en Cooper, A. (Ed.): Planning: cómo hacer el planeamiento estratégico de las comunicaciones. Buenos Aires: Thomson Learning Argentina, pp. 231-241. 
Saunders, J. (2004): “Drowing in choice: the revolution”, Market Leader, Spring, pp. 38-39.

Schofield, R. (1990): “The role of account planning in the direct-marketing agency”, Journal of Advertising Research, 30, 5, October-November, pp. 61-66. http://www.mshmgi.com/glossary,M,Media+Neutral+Planning.html (Fecha de consulta $11 / 09 / 08)$.

Shaver, M. A. (1995): Make the sale! How to sell media with marketing. Chicago: The Copy Workshop.

Stabiner, K. (1993): Inventing desire. Inside Chiat/Day: the hottest shop, the coolest players, the big business of advertising. New York: Simon \& Schuster.

Tapp, A. (2005): “Media neutral planning -a strategic perspective”, Journal of Database Marketing \& Customer Strategy Management, 12, 2, pp. 133-141.

Taylor, J. (2005): Space race. An inside view of the future of communications planning. New York: John Wiley \& Sons Ltd.

The Commoditization of Brands and Its Implications for Marketers, Copernicus Marketing Consulting. Disponible en : http://www.copernicusmarketing.com/about/branding_study.shtml (Fecha de consulta 14/11/08).

Tilley, A. (2000): “The strategic importance of media”, en Butterfield, L., (Ed.): Excellence in advertising. IPA, Oxford: Butterworth Heinemann, pp. 193-211.

Tungate, M. (2007): Ad Land. A global history of advertising. London: Kogan Page.

Victoria Mas, J.S. (2005) (Coord.): Reestructuras del sistema publicitario. Barcelona: Ariel Comunicación.

Weichselbaum, H. (2008) (Ed.): Readings in Account Planning. Chicago: The Copy Workshop.

Whelan, R. (1978): “Account planners: myth or the formula for agency success?”, Campaign, 2 June, pp. 39-40.

White, R. (2004): “Knowledge into insight: market segmentation and beyond”, en Saunders, J. (Ed.): The Communications Challenge: a practical guide to media neutral planning, London: The Account Planning Group, pp 23-52.

(2008): “Account planning: 40 years of the revolution”, Admap, April, pp. 20-25.

Zambardino, A., Goodfellow, J., (2003): "Account planning in the new marketing and communications environment (has the Stephen King challenge been met?)", en Marketing Intelligence and Planning, 21, 7, pp. 425-434. 
